Iraqi Journal of Information and Communications Technology(IJICT)

Conference Series: The $1^{\text {st }}$ Conference of Applied Researches

in Information Engineering(ARIE2021), 2021

ISSN:2222-758X

e-ISSN:2789-7362

\title{
BLIND RESTORATION USING CONVOLUTION NEURAL NETWORK
}

\author{
Meryem H. Muhson ${ }^{1}$, Ayad A. Al-Ani ${ }^{2}$ \\ 1,2 College of Information Engineering, Al-Nahrain University, Baghdad, Iraq \\ meryemconan95@gmail.com ${ }^{1}$, ayad.abdulaziz@ coie-nahrain.edu.iq ${ }^{2}$ \\ Received:5/5/2021, Accepted:6/6/2021
}

\begin{abstract}
Image restoration is a branch of image processing that involves a mathematical deterioration and restoration model to restore an original image from a degraded image. This research aims to restore blurred images that have been corrupted by a known or unknown degradation function. Image restoration approaches can be classified into 2 groups based on degradation feature knowledge: blind and non-blind techniques. In our research blind algorithm has been adopted. For single image super-resolution, a deep learning method with super-resolution has been proposed. This approach can learn an end-to-end mapping between low-resolution images and highresolution images directly. The mapping is expressed by a deep convolutional neural network (CNN). The proposed restoration system must overcome and deal with the challenges that the degraded images have unknown kernel blur, to deblur degraded images as an estimation from original images with a minimum rate of error. Comparing with the traditional bicubic method in super-resolution, our proposed system (FSRCNN) has better performance in de-blurring with fast training speed and good results according to what the results showed for the metrics used to measure the performance of our system. The paper's contribution is restoring blurred images corrupted by a known or unknown degradation function.
\end{abstract}

keywords: Image restoration, Blind deconvolution, Deep learning, Deep convolutional neural networks, Image super-resolution.

\section{INTRODUCTION}

Image restoration can be defined as one of the conventional problems in the low-level vision that was commonly studied in the literature. In general, image restoration represents an operation that takes a corrupted image then attempting at the estimate the original image. The restoration of the image is divided into two phases, these phases are: the degradation phase and restoration phase, Fig. 1 shows these two phases [1]:

1) Degradation phase: During this phase, the original image is degraded with blurring function and the extra noise function. The resultant image of this process is called a degraded image.

2) Restoration phase: At this phase, we applied the restoration algorithm to the degraded image, to estimate the restored image.

The degradation model is usually modeled as [1]:

$$
g(x, y)=f(x, y) \otimes h(x, y)+n(x, y)
$$

Where: $g(x, y):$ degraded (Blurred and Noisy) image, $f(x, y):$ original image (Object), $n(x, y):$ the additive white Gaussian noise, $h(x, y):$ the Blurring function, or called Point Spread Function PSF , and $\otimes:$ denotes convolution process. For more details, see [1], [2], [3] . For Blind de-convolution, in several cases, the imaging process that leads to $\mathrm{g}(\mathrm{x}, \mathrm{y})$ has not been entirely uncovered, and $\mathrm{h}(\mathrm{x}, \mathrm{y})$ has been unknown in Eq. 1. Considering degraded image, $\mathrm{g}(\mathrm{x}, \mathrm{y})$, the blind deconvolution aims at the simultaneous reconstruction of both $\mathrm{h}(\mathrm{x}, \mathrm{y})$ and unknown image $\mathrm{f}(\mathrm{x}, \mathrm{y})$ [4], [5]. Lately, Deep NNs (DNNs) showed that they have better efficiency in the tasks of computer vision and image processing, which range 
from high-level recognition, semantic segmentation to low-level de-noising and Super Resolution. Image Super-Resolution (SR) can be defined as the task of recovering a high-resolution from lower-resolution images. This problem is notable for its applications in security as well as in medical imaging, especially since image reconstruction offers a methodology for correcting imaging system imperfections. In the present study, a deep learning approach has been suggested, which can simultaneously address restoration and SR from low resolution blurred images. The proposed model has been referred to as the restoration SR CNN that is applied to the super-resolution on the blurred/noisy images with a priori unknown (i.e. blind) amount of the blurring. This model has been experimentally validated and it has been shown that the proposed architecture has been more suitable for the reconstruction of the blurred images in comparison to the results that have been obtained using the Bicubic approach [2], [4] in blind scenarios.

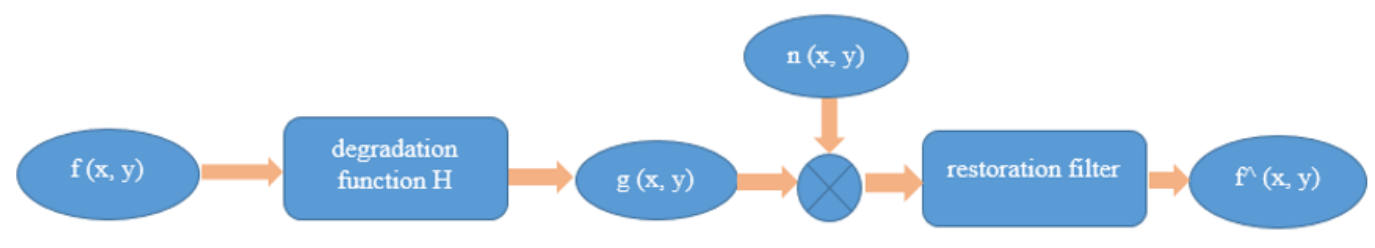

Figure 1: Represent the degradation and restoration phases

\section{RELATED WORK}

Chao Dong. et al. [7], presented an innovative deep learning method for the single image SR. They have shown that the traditional sparse-coding-based SR approaches may be carried out to a deep CNN. Kim et al. [8], present an SR approach with the use of very deep networks, through using the residual-learning and extremely high learning rates to optimize the very deep network. fast. Lin et al. [9] proposed method is Deep Convolutional Networks for SR. Based on CNN concepts, DCNSR has been modeled as 4 convolutional layers with sufficient sizes of the filter for improving the accuracy of the reconstruction over the SRCNN. Hsu et al. [10] applied a capsule neural network to CNN-based image SR approaches. CIRNN uses rich information that is contained in capsules for the direct reconstruction of images. Shankar. et al. [11], construct a model utilizing CNNs, which performed adequately as well as increases the efficiency of sharpening the blurred image and reduced the training time.

\section{SySTEM ARChiteCTURE}

The pictorial representation of our architecture is shown in Fig. 2. A brief explanation of our architecture is as follows:

1) Pre-processing steps: Normalization of the image inputs, the normalization of the data is one of the most significant steps, ensuring the fact that every one of the input parameters (in this case, pixels) has a similar distribution of the data. Which results in making the convergence more rapid throughout the training of the network [12]. The process of the normalization can be represented as:

$$
x_{n e w}=\frac{x-x_{\min }}{x_{\max }-x_{\min }}
$$


$\mathrm{x}$ : represents a set of the observed values present in $\mathrm{x} . x_{\min }:$ is the minimum values in $\mathrm{x}$, and $x_{\max }:$ is the maximum values in $\mathrm{x}$.

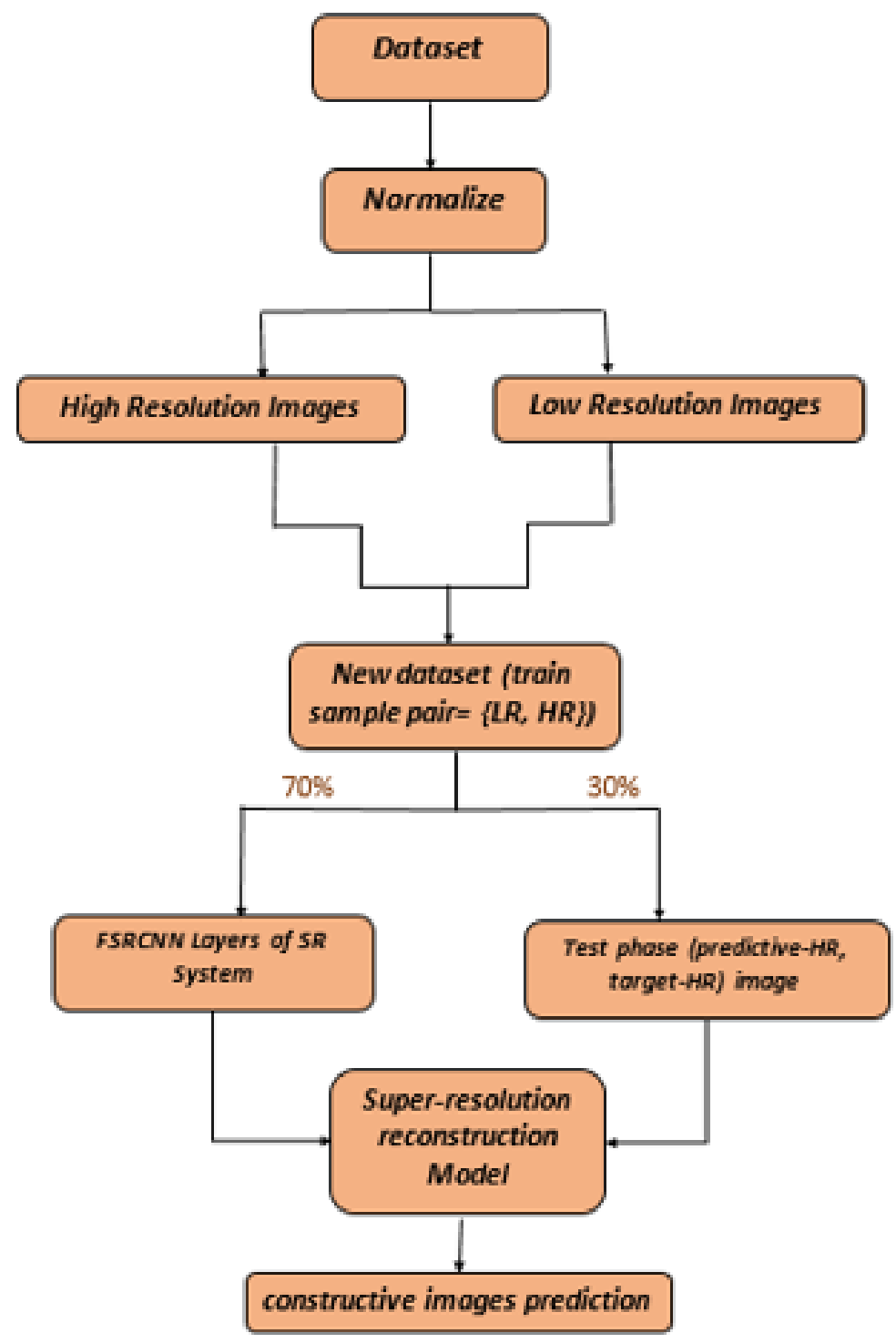

Figure 2: Block diagram of proposed system

2) create the low resolution(LR): To create a single noisy/ blurred LR sub-image (i.e. input) for the testing and training, first, HR sub-images are blurred with the use of a Gauss kernel that has been noted (by the standard deviation: $\sigma=i$ ). Second: the images undergo the scaling interpolation. The down/upscaling factor that has been utilized in the present paper is $s=4$ (the image is down-sampled and after that up-sampled by an $s=4$ factor) Resize the sub-image by the 
up/down-scaling factor, and up/down-scaled it by the same factor via bicubic interpolation [22], and the Additive White Gauss noise is added (with different value of the SNR) [13] shown in Fig. 3).

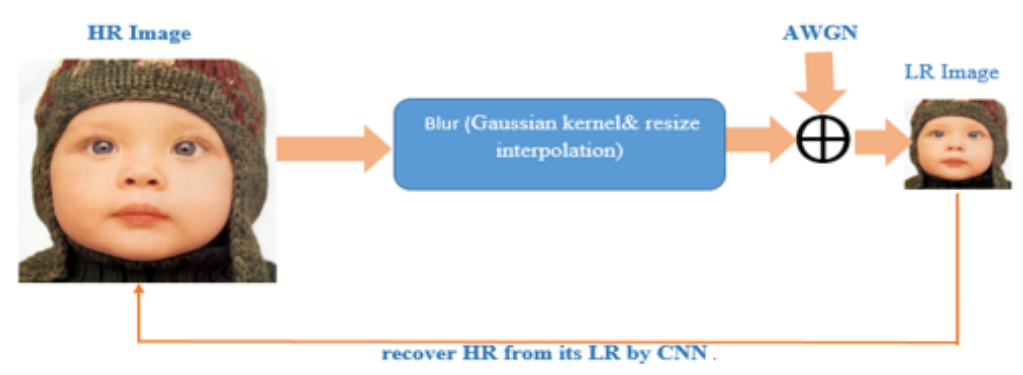

Figure 3: Overall framework of create low resolution

3) Training a network: Network Training can be defined as a procedure that includes finding the kernels in convolution layers and weights that minimize the differences between output predictions constructive images and considering the ground truth labels on the training data-set. The algorithm of backpropagation can be defined as the approach that is typically utilized for the training of the NNs, in which the algorithm of the gradient descent optimization and loss function play essential roles [14] Fig. 4 shown the main step of train process in CNN model. For the strategy of the training in the training phase, initially, the original colour image is converted into the grey-scale image through the extraction of the component of the luminance in the color space of the YCbCr. To entirely exploit available data, augmentation has been relied upon: the HR images from the training dataset are cropped randomly for obtaining $f_{\text {sub }} \times f_{\text {sub }} \times c$ pixel sub-images. Sub-images of size $f_{\text {sub }}=20$ have been employed, which is why 91 HR images may be split into 213854 training sub-images and validate sub- images 3008 with the stride 3 . The model has been trained upon the sub-images, and inference upon the entire image. In the case where the training by 91-image data-set, the size of the update has been regulated with the rate of the learning. The learning steps' size has been referred to as learning rate $\alpha$ which provides additional control over how large one makes. The convolutional layers' learning rate has been set to $10^{-3}$ and that of de-convolution layer has been $10^{-4}$. After that, throughout the algorithm of the optimization, the learning rate of all of the layers is decreased by $50 \%$.

4) Prepare a model: Divide the entire dataset into 2 parts: training and validation (i.e. testing) sets. The proposed approach is using $70 \%$ of data for the training and the rest $30 \%$ for testing (There is no optimal split percentage but the training dataset should be larger to have a better deep learning rate than the test dataset and depends upon the dataset size in our project we have 91 images for train set so we preferred to use 70:30 ratio) Ultimately, the model's accuracy on testing data-set/images can give an adequate estimation of the model's efficiency on entirely new data-set/images and to confirm the actual predictive power of SR network which has been constructed [15]. 


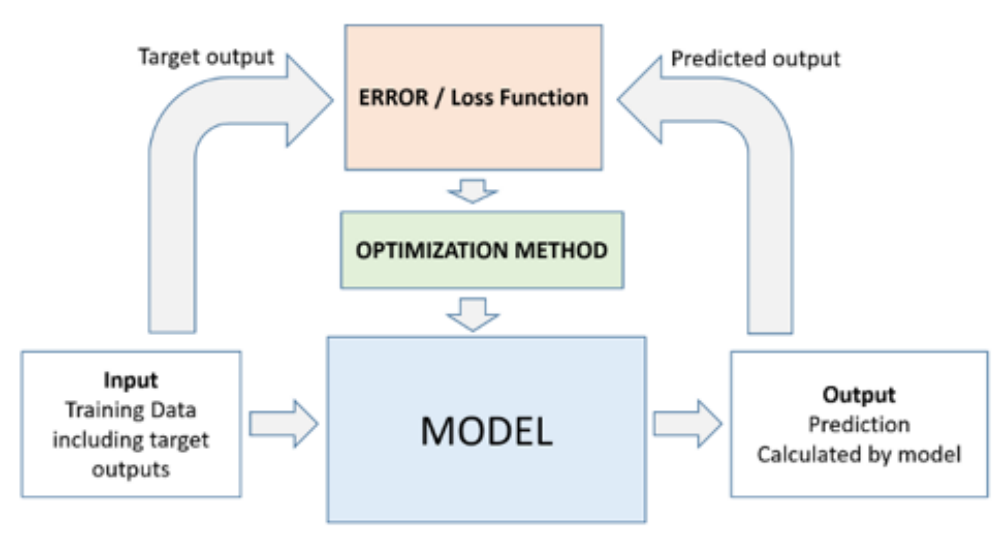

Figure 4: Main architecture of train process in CNN model

\section{Proposed CNN Algorithm}

A Fast Super-Resolution CNN (FSRCNN) model has been proposed [16], [17]. The network directly learns an end-to-end mapping between the images of high and low resolution, SRCNN has been suggested, increasing the network depth and presents a de-convolution layer for restoring the features, this layer may result in realizing conversion from the low- to the high-resolution space. This characteristic permits FSRCNN from the direct utilization of low-resolution images rather than the result of the interpolation as network input. The direct use of the LR images as the input can't only result in the reduction of the model's calculation amount, however, the accuracy of the restoration is insufficient to some extent. This structure of the network results in the direct convolution of an original small low-resolution image, feature extraction, reconstructing it with a layer of de-convolution and adding an expanding and shrinking layer and a step of Nonlinear mapping has been defined as the most significant part affecting the performance of the super-resolution for achieving high operating speed with no recovery loss [18]. It can be seen in Fig. 5 which will define the layers of it which is 6 layers. as shown in Fig. 4 that Fi is represented the number of filters used in Convolutional layers, S represents stride, $\mathrm{K}$ represents kernel size the general FSRCNN network is proposed in the first 5 layers ReLu [18], Which is the corrected linear measurement, using (A) the Activation function. It results in 0 for any negative value of the pixel; similarly, it results in an identical for any positive value of the pixels and the Transpose layer is part of the de-convolution for the up-sampling succeeded by the linear activation function [18] .

\section{Performance Evaluation and data-set}

The training data-set is including 91 images that have been obtained from Yang et al. [20]. The testing data sets have been represented as "Set 5" (i.e. 5 images) [19] and "Set 14" (14images) [21]. This choice of training and testing data has been made for allowing a fair comparison to where they have been utilized [14] . To find the efficiency of this implemented model, we use different metrics to measure the degree of similarity between original and input noisy/blur images. three metrics had employed which is PSNR (peak signal to noise ratio) and SSIM (structural similarity), which helps to find the 
structural similarity between 2 images, and MSE (mean square error) [8]. Computation Time the Python implementation of FSRCNN uses Keras Optimizing a Keras neural network with the Adam optimizer results in a model that has been trained to make predictions accurately. To render the FSRCNN training more computationally efficient, the training time of FSRCNN in this implementation with 100 epochs (number of training iterations) was one hour and 33.33 minutes with AMD Radeon R7 M265 Series. Results of Our Network: to fully evaluate the efficiency of the FSRCNN on the blurred images had been tested blind scenarios that have been trained on blurred images $\mathrm{N}(0, \sigma)$ we choose $\sigma$ in Gaussian blur that vary between (1-4). AWGN with SNR varying between $(10,50)$. Testing has been carried out on images that have a variety of blur/noise levels. The quantitative measurement of bicubic interpolated image and FSRCNN reconstructed image with the ground truth image. It may be seen that FSRCNN reconstructed image has better quality than the bicubic interpolated image for all standard deviation $(\sigma)$ 1, 2 and signal to noise ratio (SNR) 1,5,10,50.

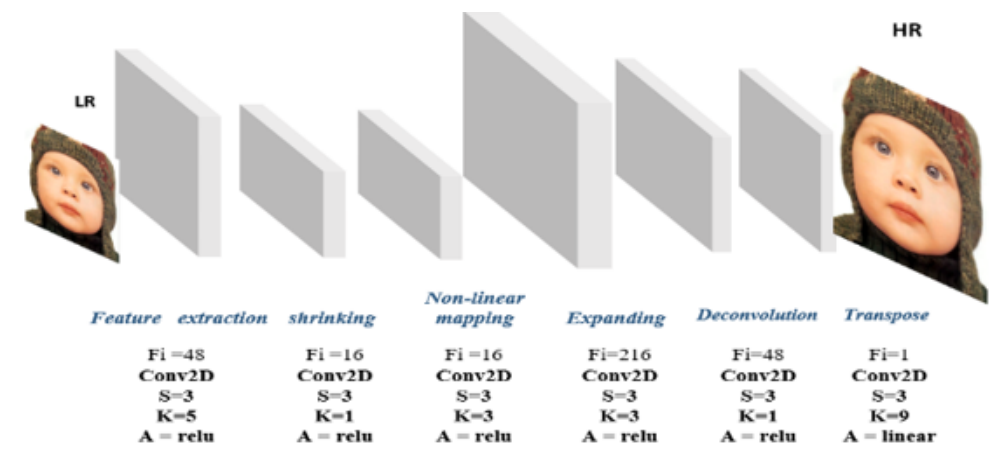

Figure 5: The overall network of FSRCNN

TABLE I

The Results of SET14 and SET5 Testing The Network with Gaussian Blur with $\sigma=1$ and different SNR of AWGN

\begin{tabular}{|c|c|c|c|c|c|c|c|}
\hline \multirow{2}{*}{ Dataset } & $\begin{array}{c}\text { standard } \\
\text { deviation } \sigma=1\end{array}$ & \multicolumn{3}{|c|}{ Bicubic Method } & \multicolumn{3}{c|}{ FSRCNN(our system) } \\
\cline { 2 - 8 } & SNR & PSNR & SSIM & MSE & PSNR & SSIM & MSE \\
\hline \multirow{3}{*}{ Set14 } & 10 & 29.2008 & 0.8836 & 104.9790 & 29.2435 & 0.8884 & 100.7938 \\
\cline { 2 - 8 } & 50 & 29.2435 & 0.8847 & 103.5759 & 29.2969 & 0.8953 & 100.8634 \\
\hline \multirow{2}{*}{ Set5 } & 10 & 33.0757 & 0.9372 & 46.0084 & 33.3448 & 0.9382 & 42.8600 \\
\cline { 2 - 8 } & 50 & 33.2654 & 0.9382 & 47.4284 & 33.4913 & 0.9395 & 40.9649 \\
\hline
\end{tabular}

TABLE II

The Results of SET5 and SET14 Testing The Network with Gaussian Blur with $\sigma=2$ and Different SNR of AWGN

\begin{tabular}{|c|c|c|c|c|c|c|c|}
\hline \multirow{2}{*}{ dataset } & $\begin{array}{c}\text { standard } \\
\text { deviation } \sigma=2\end{array}$ & \multicolumn{3}{|c|}{ Bicubic Method } & \multicolumn{3}{c|}{ FSRCNN(our system) } \\
\cline { 2 - 8 } & SNR & PSNR & SSIM & MSE & PSNR & SSIM & MSE \\
\hline \multirow{2}{*}{ Set14 } & 10 & 28.4257 & 0.8641 & 122.5327 & 29.2435 & 0.8884 & 100.7938 \\
\cline { 2 - 8 } & 50 & 28.334 & 0.8604 & 125.6115 & 29.4465 & 0.8993 & 100.5006 \\
\hline \multirow{2}{*}{ Set5 } & 10 & 32.0515 & 0.9261 & 61.9286 & 33.0757 & 0.9382 & 46.0084 \\
\cline { 2 - 8 } & 50 & 32.0434 & 0.9245 & 62.6940 & 33.2654 & 0.9394 & 46.0062 \\
\hline
\end{tabular}




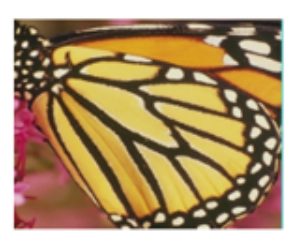

Orizinal (SNR, o)

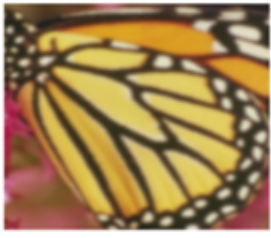

$\operatorname{LR}(10,2)$

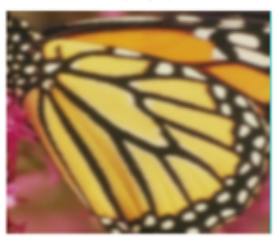

$\operatorname{LR}(50,2)$

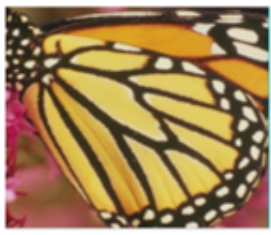

$\operatorname{FSRCNN}(10,2)$

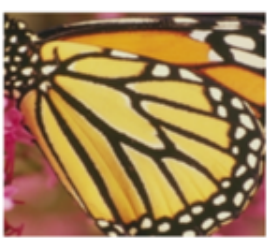

$\operatorname{FSRCNN}(50,2)$

Figure 6: Butterfly image from Set5

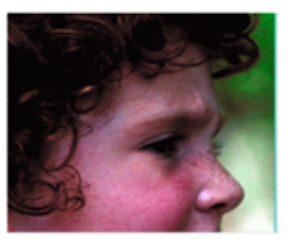

Original (SNR, б)

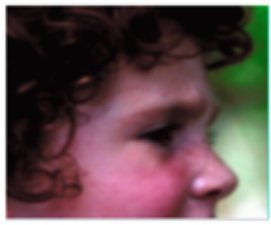

$\operatorname{LR}(10,2)$

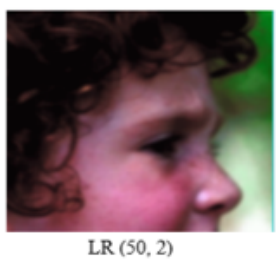

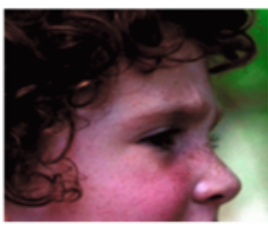

FSRCNN (10, 2)

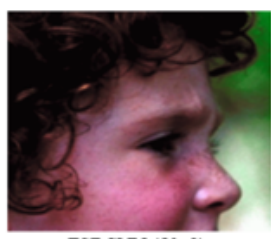

$\operatorname{FSRCNN}(50,2)$

Figure 7: Face image from Set14

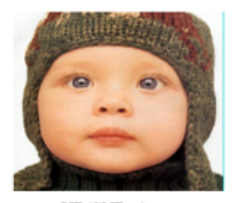

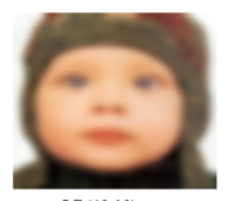

LR(40,10)

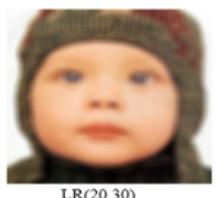

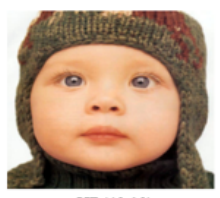

HR(40,10)

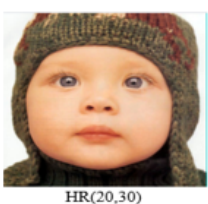

Figure 8: Baby image from Set5 
Iraqi Journal of Information and Communications Technology(IJICT)

Conference Series: The $1^{\text {st }}$ Conference of Applied Researches

in Information Engineering(ARIE2021), 2021

ISSN:2222-758X

e-ISSN:2789-7362

\section{Conclusion}

We proposed an image blind restoration method based on the deep learning method for image SR. it is seen that the restoration of the image may be re-formulated to a deep CNN. The suggested method, the FSRCNN network is used as the framework of the super-resolution reconstruction method. Our experimental study with various degrees of noise and Gaussian blur demonstrated that the proposed network deeper architecture had performed more sufficient scenarios of the blind testing. The proposed approach has been compared to the conventional bicubic method. Our goal provided better-reconstructed image quality have been achieved.

\section{REFERENCES}

[1] Ayad A. Al-Ani, "Restoration of Atmospherically Degraded Images" , Department of Physics, College of Science, Baghdad University, Baghdad,Iraq, $\mathrm{PhD} 1995$.

[2] A. H. Sheer and A. A. Al-Ani, "The Effect of Regularization Parameter within non-Blind Restoration Algorithm using Modified Iterative Wiener Filter For Medical Images" , 1st Annual International Conference for Information and Science, AICIS, IEEE recorded No. 46152, 19-21 Nov. ,2018.

[3] A. A. Al-Ani, "An Adaptive Iterative Image Restoration Using Projection onto Convex Sets Method" , The fourth Saudi Technical Conference and Exhibition, Vol. IV, pp. 272-278, 2006.

[4] Marhaba, B, Zribi, M, \& Khodar, W, "Image Restoration Using A Combination of Blind and Non-Blind Deconvolution Techniques" , International Journal of Engineering Research and Science,No. 5, pp. 225-239, 2016.

[5] Woods, R. E,Rafael C. Gonzalez,2002.

[6] R. Keys, "Cubic Convolution Interpolation for Digital Image Processing" , In: IEEE Transactions on Acoustics, Speech, and Signal Processing 29.6 , pp. 1153-1160, 1981.

[7] Dong, C, Loy, C. C, He, K, \& Tang, X, "Image Super-Resolution Using Deep Convolutional Networks" , IEEE transactions on pattern analysis and machine intelligence, Vol. 38, No. 2, pp. 295-307, 2015.

[8] Kim, J, Lee, J. K, \& Lee, K. M, "Accurate Image Super-Resolution Using Very Deep Convolutional Networks" , In Proceedings of the IEEE conference on computer vision and pattern recognition, pp. 1646-1654, 2016.

[9] Lin, G, Wu, Q, Huang, X, Qiu, L, \& Chen, X,2017, August, "Deep Convolutional Networks-Based Image Super-Resolution" , In International Conference on Intelligent Computing, pp. 338-344, Springer, Cham.

[10] Hsu, J. T, Kuo, C. H, \& Chen, D. W, "Image Super-Resolution Using Capsule Neural Networks" , IEEE Access, No. 8, pp. 9751-9759, 2020.

[11] Shankar, R. S, Mahesh, G, Murthy, K. V. S. S, \& Rajanikanth, J, "A Novel Approach for Sharpening Blur Image Using Convolutional Neural Networks", Journal of Critical Reviews, Vol. 7, No. 7, pp. 139-148, 2020.

[12] Gopal Krishna Patro, S, \& Sahu, K. K, "Normalization: A Preprocessing Stage" , arXiv e-prints, p. 1503, pp. 62-64, 2015.

[13] Singh, P, \& Shree, R, "A Comparative Study to Noise Models and Image Restoration Techniques", International Journal of Computer Applications, Vol. 149, No. 1, pp. 18-27, 2016.

[14] Cui, N, "Applying Gradient Descent in Convolutional Neural Networks" , In Journal of Physics: Conference Series, Vol. 1004, No. 1, p. 012027, pp. 1-8 April, IOP Publishing, 2018.

[15] Xu, Y, \& Goodacre, R, "On Splitting Training and Validation Set: A Comparative Study of Cross-Validation, Bootstrap and Systematic Sampling for Estimating the Generalization Performance of Supervised Learning", Journal of Analysis and Testing, Vol. 2, No. 3, pp. $249-262,2018$.

[16] Zhang, H, Wang, P, Zhang, C, \& Jiang, Z, "A Comparable Study of CNN-Based Single Image Super-Resolution for Space-Based Imaging Sensors" , Sensors, Vol. 19, No. 14,p. 3234,pp. 1-18, 2019.

[17] Anwar, S, Khan, S, \& Barnes, N, "A Deep Journey Into Super-Resolution: A Survey" , ACM Computing Surveys (CSUR), Vol. 53, No. 3, pp. 1-34, 2020.

[18] Dong, C, Loy, C. C, \& Tang, X, "Accelerating the Super-Resolution Convolutional Neural Network" , In European conference on computer vision, pp. 39-407, Springer, Cham, October, 2016.

[19] Bevilacqua, M, Roumy, A, Guillemot, C, \& Alberi-Morel, M. L, "Low Complexity Single Image Super Resolution Based on Nonnegative Neighbor Embedding", 2012.

[20] Yang, J, Wright, J, Huang, T. S, \& Ma, Y, "Image Super-Resolution Via Sparse Representation" , IEEE transactions on image processing, Vol. 19, No. 11, pp. 2861-287, 2010.

[21] Zeyde, R, Elad, M, \& Protter, "On Single Image Scale-Up Using Sparse-Representations" , In International conference on curves and surfaces, pp. 711-730, Springer, Berlin, Heidelberg, June, 2010.

[22] Wittman, T, "Mathematical Techniques for Image Interpolation" , Report Submitted for Completion of Mathematics Department Oral Exam, Department of Mathematics, University of Minnesota, USA, 2005. 